UCRL-JC-120894

PREPRINT

\title{
Application of 3D X-Ray CT Data Sets to Finite Element Analysis
}

\author{
P-L. Bossart \\ H. E. Martz \\ H. R. Brand \\ K. Hollerbach
}

This paper was prepared for submittal to the 22 Quantitative Non-destructive Evaluation Conference Seattle, WA

July 30-August 4, 1995

August 31, 1995

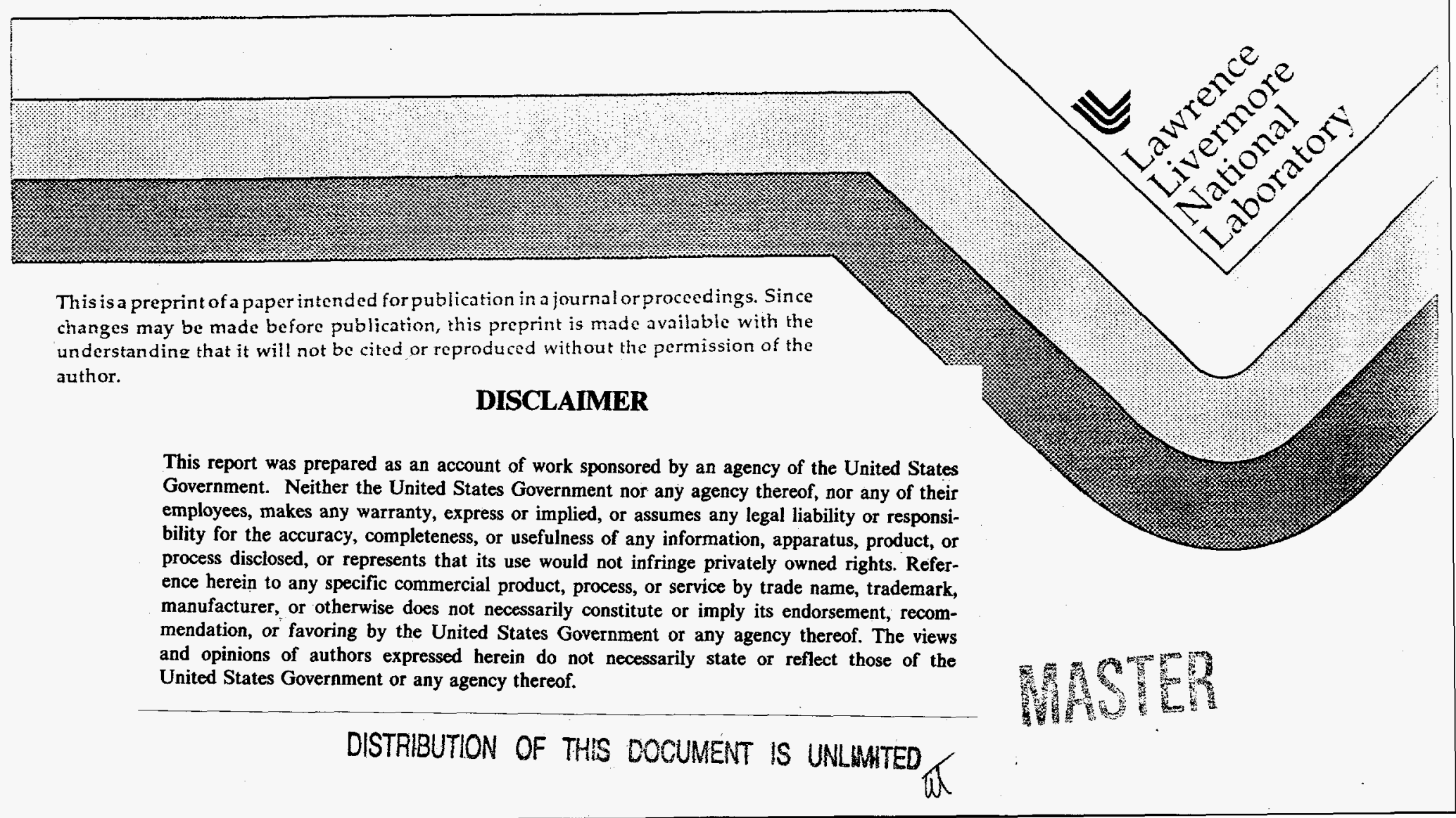




\section{DISCLAIMER}

This document was prepared as an account of work sponsored by an agency of the United States Government. Neither the United States Government nor the University of California nor any of their employees, makes any warranty, express or implicd, or assumes any legal liability or responsibility for the accuracy, completeness, or usefulness of any information, apparatus, product, or process disclosed, or represents that its use would not infringe privately owned rights. Reference herein to any specific commercial product, process, or service by trade name, trademark, manufacturer, or otherwise, does not necessarily constitute or imply its endorsement, recommendation, or favoring by the United States Government or the University of California. The views and opinions of authors expressed hercin do not necessarily state or reflect those of the United States Covernment or the University of California, and shall not be used for advertising or product endorsement purposes. 


\section{DISCLAIMER}

Portions of this document may be illegible in electronic image products. Images are produced from the best available original document. 


\title{
APPLICATION OF 3D X-RAY CT DATA SETS TO FINITE ELEMENT ANALYSIS*
}

\author{
P-L Bossart, H.E. Martz, H.R. Brand and K. Hollerbach \\ Lawrence Livermore National Laboratory, L-333 \\ 7000 East Avenue \\ Livermore, CA 94550 \\ Email: bossart1 @llnl.gov
}

\begin{abstract}
Finite Element Modeling (FEM) is becoming more important as industry drives toward concurrent engineering. A fundamental hindrance to fully exploiting the power of FEM is the human effort required to acquire complex part geometry, particularly as-built geometry, as a FEM mesh. Many Quantitative Non Destructive Evaluation (QNDE) techniques that produce three-dimensional (3D) data sets provide a substantial reduction in the effort required to apply FEM to as-built parts. This paper describes progress at LLNL on the application of 3D X-ray computed tomography (CT) data sets to more rapidly produce high-quality FEM meshes of complex, as-built geometries. Issues related to the volume segmentation of the 3D CT data as well as the use of this segmented data to tailor generic hexahedral FEM meshes to part specific geometries are discussed. The application of these techniques to FEM analysis in the medical field is reported here.
\end{abstract}

\section{INTRODUCTION}

Finite Element Modeling is widely used by the research community and industry in order to grasp a better understanding of real-world applications through computer simulations. This engineering tool is becoming more important as industry drives towards concurrent engineering. A Finite Element Analysis (FEA) is typically made up of three steps, namely the creation of a mesh, the computer simulation, and the post-processing of the results. A fundamental hindrance to fully exploiting the power of FEM is the human effort required to acquire complex part geometries. This preprocessing can represent up to $80 \%$ of an engineer's time. Therefore, the need to minimize the amount of interactivity cannot be over stressed. Besides the need for a speed-up, the meshing should be based upon actual "as-built" geometries, which should be preferred to as-designed geometries from $C A D$ models or generic descriptions. A CAD model may not accurately account for all the changes to the initial design that take place during the manufacturing process. $A C A D$ model may simply not be available if the manufacturer has gone bankrupt or is part of the competition. In biomechanics applications, the generic descriptions of human parts are not precise enough, which limits the use of Finite Element Models.

X-ray CT allows one to understand the spatial arrangement of the parts inside of a volume, in contrary to laser range-finders or other dimensional measurement devices. Recent progress in data storage and reconstruction time make it possible to rapidly acquire large data sets. X-ray CT has thus become a key modality for extracting 3D geometries. References [1] and [2] describe the application of CT to CAD modeling, reverse

\footnotetext{
* Work performed under the auspices of the U.S. Department of Energy by the Lawrence Livermore National Laboratory under contract No. W-7405-ENG-48.
} 
engineering and rapid prototyping. In this paper, we focus on the use of 3D X-ray CT data sets to tailor accurate hexahedral meshes for dynamic finite elements simulation.

The organization of the paper will closely match the dataflow presented in Figure 1. We will first describe the CT area-detector scanner used to acquire the $3 D$ images. Issues related to preprocessing of raw CT data and tomographic reconstruction will then be discussed. We will show that despite promising results using advanced mathematical morphology techniques, a human interaction is necessary to check the accuracy of the segmentation. The need for a fully $3 \mathrm{D}$ segmentation in order to solve ambiguities will be mentioned. Details regarding the surface extraction step will be presented. We will discuss the trade-off between accuracy and smoothness of the extracted polygonal surface. The use of templates for the creation of a volumetric hexahedral mesh will be explained next, as well as the challenges that remain to be solved, e.g. when the surfaces are highly asymmetrical. After a summary of our approach, furure developments will be described. Throughout this paper, we will present the results obtained in a biomechanics application, where we aim at building Finite Element Models of a hand. However, the techniques described and the expertise gained will be applied in the future in industrial applications.

Before presenting more precisely each of these steps, let us point out that this work stems from a collaboration between several groups at LLNL, and that all the scanners, image processing and FEM software are available on-site. Thus, rather than optimizing each step of the dataflow independently from the others, we can in the contrary adjust the results and the techniques used in order to optimize the dataflow as a whole.

\section{DATA ACQUISITION AND TOMOGRAPHIC RECONSTRUCTION}

Typically, the scanners used in the medical field have a spatial resolution of about 1 $\mathrm{mm}$; the energy is also about $140 \mathrm{kVp}$. Several industrial scanners have been designed and constructed at the Lawrence Livermore National Laboratory. In our experiments we used the PCAT scanner, which can be reconfigured to handle objects of various sizes and attenuations. The pixel size of the PCAT scanner is $150 \mu \mathrm{m}$, and is equal to the distance between CT slice planes. Thus, PCAT makes it possible to acquire high-spatial resolution isotropic volume elements (voxels). PCAT uses a $450 \mathrm{kVp} \mathrm{X}$-ray source and a scintillating screen lens coupled to a 14-bit camera. This $2 \mathrm{D}$ detector helps reduce the scanning time by acquiring multiple slice planes simultaneously. Typically, we used 720 angles to build a complete set of projections. As the size of the raw ciata is on the order of one Gigabyte, the reconstruction step is the most computationally-intensive and is performed on a Silicon Graphics Onyx using a parallelized Convolution Bask-Projection (CBP) algorithm. This fan-beam approximation to a cone-beam geometry only holds since the field of view was limited to a half-cone angle of 2 degrees.

Several experiments were performed in order to assess the effects of data preprocessing and volume reconstruction from the segmentation point of view. Removing the bad pixels which appear when a photon hits the CCD directly made the segmentation significantly easier. The correction for beam-hardening also proved extremely useful. The beam-hardening effect stems from the poly-chromaticity of the X-ray source.

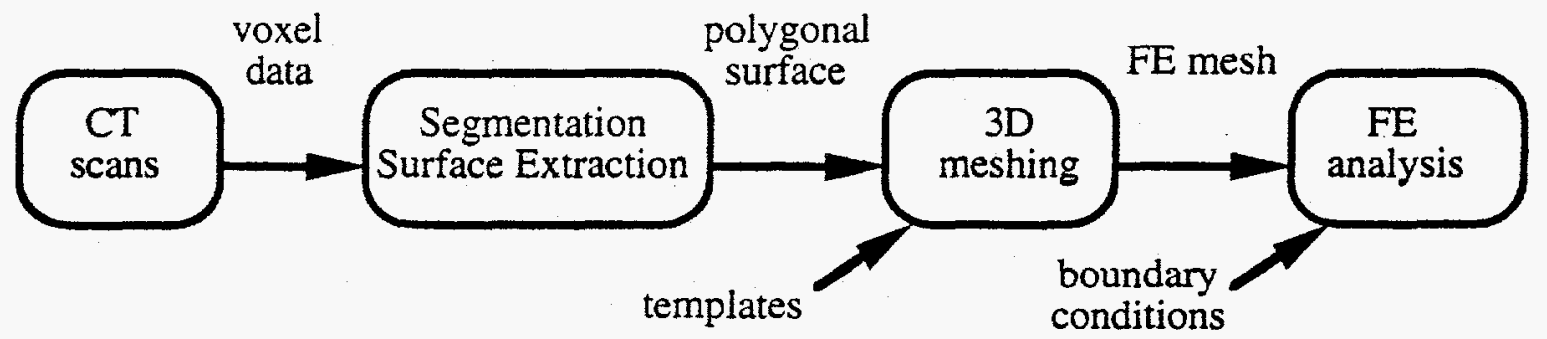

Figure 1: General dataflow required to mesh 3D CT data sets 
Since low-energy photons are more likely to be absorbed than high-energy ones, "cupping" artifacts occur in the reconstructed images. A correction of the beam-hardening effects simplified the segmentation dramatically. Results for three preprocessing approaches are shown in Figure 2. The image on the left corresponds to a reconstruction after correction for beam-hardening. In addition, the reconstructed results after a deconvolution (middle image) and a ring removal correction (right image) are shown. Although these last two preprocessing approaches yield better-looking reconstructions, they did not help improve the segmentation significantly over the beam-hardening correction approach.

Theoretical and practical reasons make it necessary to filter the projections before back-projection. Traditionally, the cut-off frequency is chosen within the interval 0.4-0.45, as these values help reconstruct visually-good images while reducing the aliasing problems. However, it is known that the eye is not sensitive to variations in the contrast which can make a segmentation algorithm fail. Several experiments were performed with different cutoff frequencies; a value of 0.3 produced the best result during the segmentation. Although the slices look blurred, they contain less variations of the contrast and less noise that can fool the segmentation algorithm.

\section{SEGMENTATION}

The interactive segmentation package VOXELMAN' was used in order to segment the 3D images. VOXELMAN makes it possible to visualize three orthogonal views of the 3D CT data set, as well as to perform a histogram-based thresholding. After several morphological operations and a connected components analysis, the segmentation can be rendered in 3D. VOXELMAN allows the user to interactively remove any spurious labels to obtain an accurate segmentation. However, this type of segmentation is tedious since it requires a large amount of human interaction. In addition to the need for an automatic procedure, it also appears necessary to take into account the structure of the materials to be segmented. Indeed, in our biomechanics applications, the bones are not homogeneous materials; as it can be seen in Figure 2, the trabecular structure of the bones creates a texture. Moreover, the bone marrow and the flesh have almost the same attenuation values. Choosing a threshold in a robust way is almost impossible because of these two problems.

We first assessed the performance of edge detectors [3], which detect and localize sharp boundaries, in order to automate the segmentation process. However the texture creates a large number of spurious edges, which make this approach not reliable.
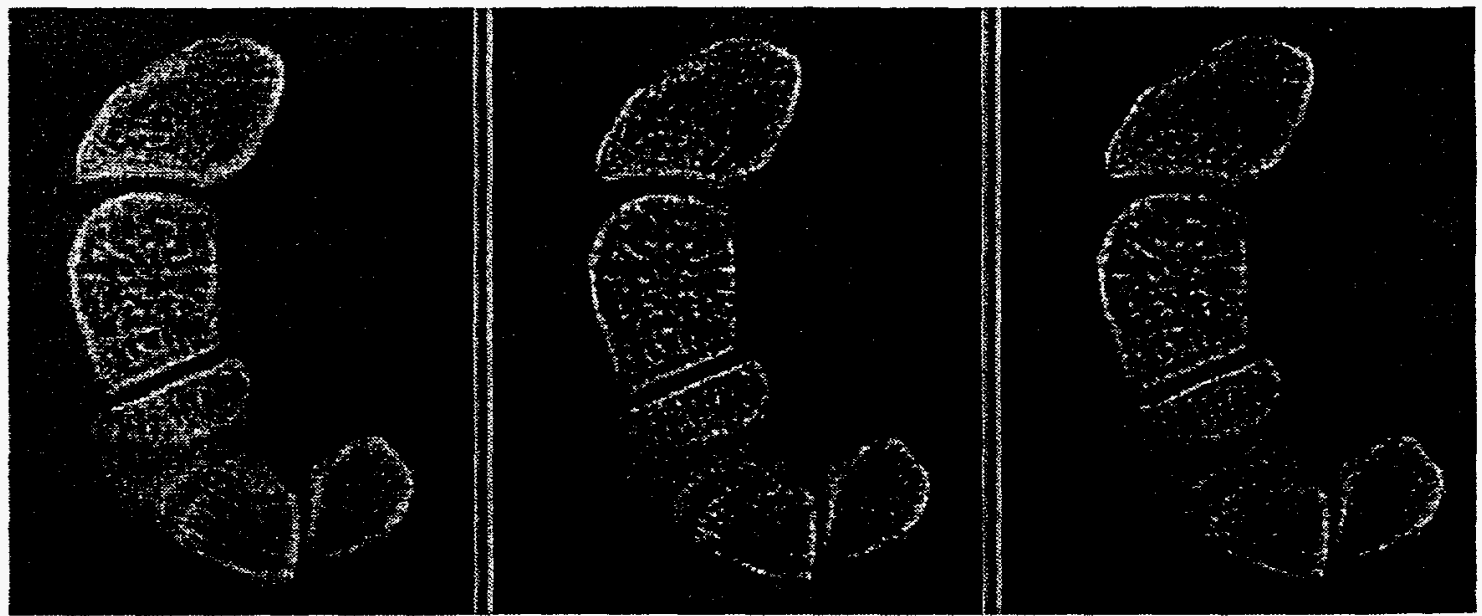

Figure 2: Reconstructed images of a hand cross-section obtained with different preprocessing approaches (see text for details).

\footnotetext{
' Public domain software, University of Hamburg, Germany.
} 
In the contrary, an efficient region-based algorithm based on advanced morphological operations yielded accurate results. A gray-scale dilation on a large neighborhood followed by a gray-scale reconstruction [4] helps remove the texture and create two separate regions (cf. Figure 3b). The initial histogram is unimodal, which explains the problems encountered with a threshold-based technique. After the gray-scale reconstruction, the histogram becomes bimodal; a threshold can then be chosen, which produces an initial coarse segmentation (cf. Figure $4 \mathrm{~b}$ ). The extraction of the watershed lines [5] using as inputs both the initial coarse segmentation and the gradient image (cf. Figure 4c) yields an accurate solution as shown in Figure 4d. It is useful to note that the watershed lines are located by construction on gradient peaks and hence on sharp boundaries. Although this approach helped limit the amount of human interaction, the results in Figure 4 clearly show its limits when applied to the hand data set. In this example, we cannot resolve two (bottom left bones) of the five finger bones during the thresholding step. After computation of the watershed lines, these two bones result in one marker (the gray marker in Figure 4d). In other words, while this morphological approach was satisfactory in most cases, there exist a number of cases in which a human interaction is needed to correct either the initial segmentation or the end-result. Alternatively, instead of processing the data as a set of slices, using a 3D neighborhood would allow us to discard most of the spurious solutions. We are currently working on the design of a fully-3D segmentation algorithm.

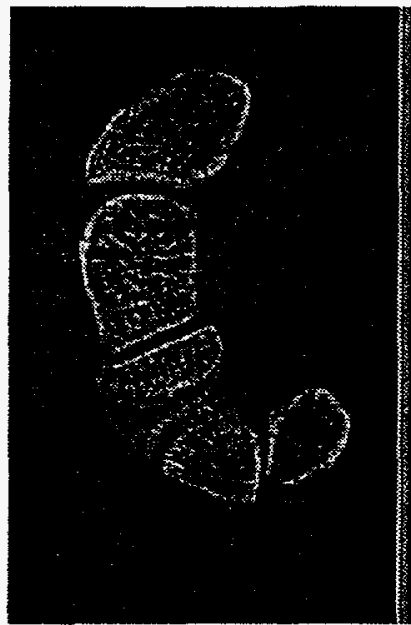

(a)

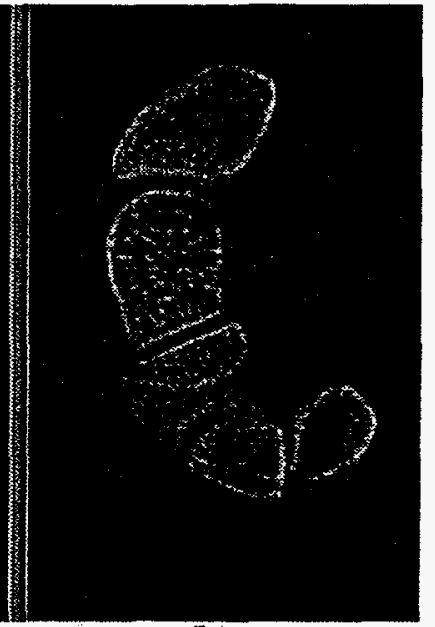

(b)

Figure 3: (a) Representative CT image of a hand; (b) Result after gray-scale mathematical morphology image reconstruction.

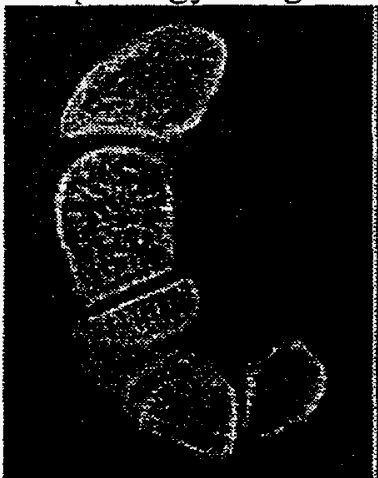

(a)

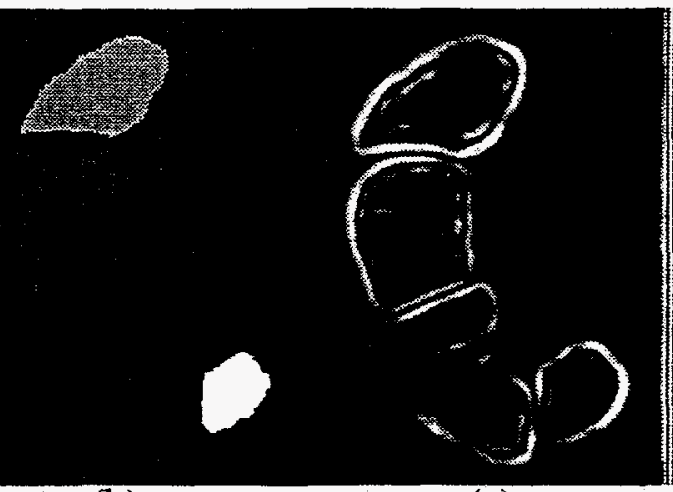

(b)

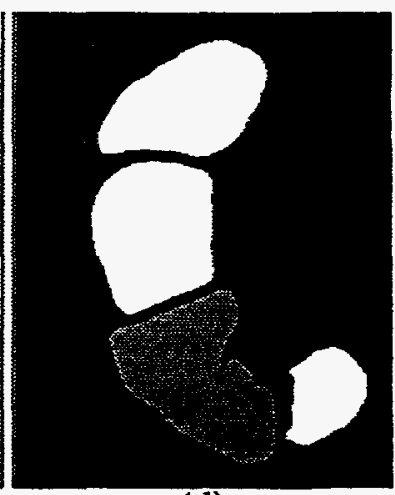

(d)

Figure 4: Region-based segmentation using the watershed concept: (a) Original image; (b) Markers extracted after thresholding of the image in Fig. 3b; (c) Gradient of the image in (a); (d) Results of region-growing algorithm. 


\section{SURFACE EXTRACTION}

Before the meshing can take place, the segmented binary volume needs to be processed in order to reduce the size of the data. Typically, a polygonal surface is extracted by scanning the volume and dividing the boundary voxels into triangular patches. Some pathological cases need to be handled with care so that the resulting surface retains the topology of the segmented volume and does not present any inconsistencies or holes. The Marching Cubes algorithm [6] provides an efficient way of extracting such a surface mesh, which can be used directly by the meshing software. However, the number of vertices required to represent a complex geometry is on the order of a million. A decimation of the polygonal surface using the Decimate ${ }^{2}$ package helped remove around $70 \%$ of the vertices, for example by replacing small triangles in flat regions by larger ones. Alternatively, the segmented volume may be represented by a set of bidimensional contours that need to be linked together so as to form a 3D surface. The Nuages ${ }^{3}$ package relies on Delaunay triangulations to perform the surface reconstruction. We have found that the algorithm used performed very well in all cases, even in the case of branching or merging structures, while requiring no interaction or choice of parameters. Figure 5 shows a $3 \mathrm{D}$ rendering of the surface of a metacarpal bone extracted from the CT data set.

Another problem comes from the discrete nature of the data. Typically, the aspect of the surface is jagged, even for noiseless data. Thus, the mesh needs to be smoothed while remaining close to the acquired data. These two conditions cannot be fulfilled simultaneously, and a trade-off needs to be found. In biomechanics applications, most surfaces are expected to be smooth, so that we can enforce the smoothness directly. In an industrial application however, the comers and angles are likely to represent the main features of a part; they should not be smoothed at all. These two remarks show clearly that no general method can be applied in all cases to smooth a polygonal mesh. On the contrary, the requirements of each application need to be taken into account.

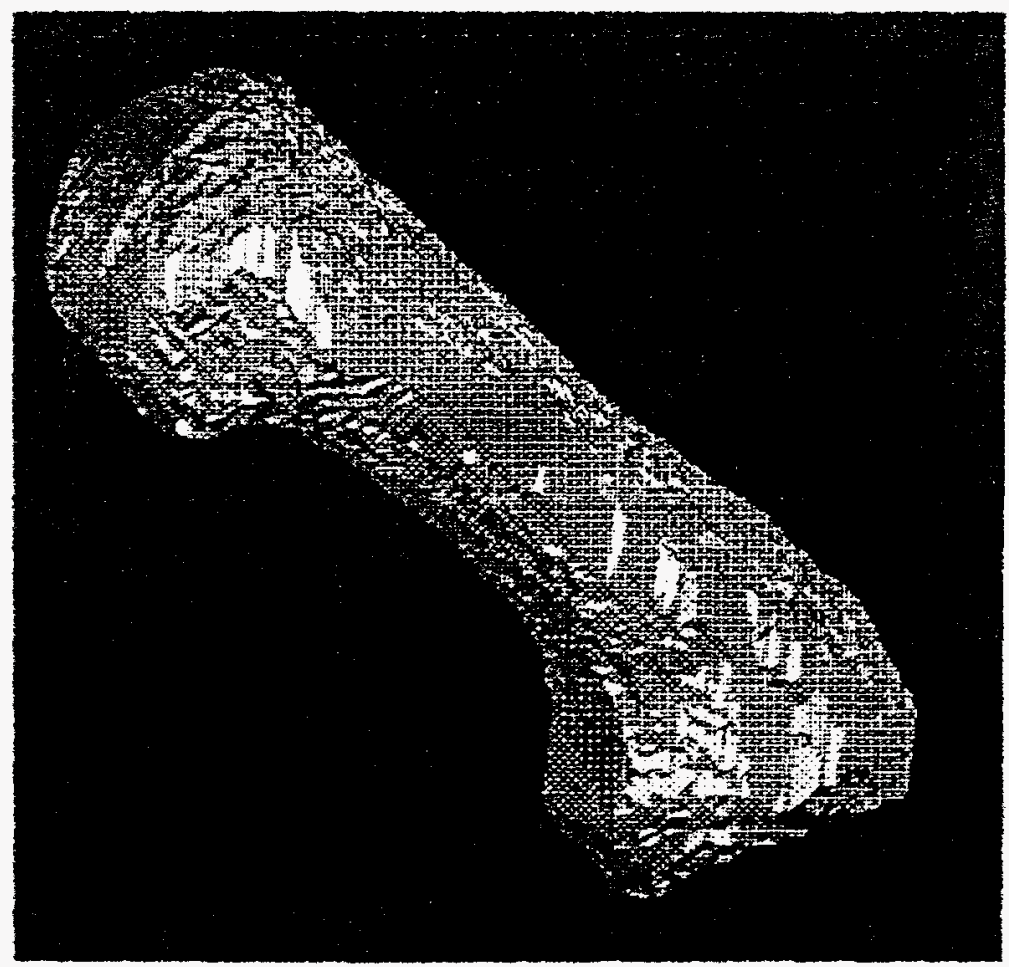

Figure 5 : 3D rendering of metacarpal bone extracted from 3D CT data.

\footnotetext{
2 Licensed from General Electric Corporate Research Development (cf. reference [7] for details)

${ }^{3}$ Public domain software, INRIA (http://www.inria.fr/prisme/personnel/geiger/nuages.html or cf. [8]).
} 


\section{CREATION OF A VOLUMETRIC HEXAHEDRAL MESH}

Let us point out that most of the papers published on automatic mesh generation focus on volumetric tetrahedral meshes. The algorithms used generally rely on a subdivision algorithm of the volume, such as in the octree approach [9]. A mesh is then built by triangulating each of the cells of the volume. Changing slightly the coordinates of the vertices helps smooth the mesh and improve its quality.

However, tetrahedral meshes are not suited for the dynamic simulations required in our applications. Structural engineers tend to prefer hexahedral meshes, which help speedup the convergence of the numerical algorithms. A tetrahedral mesh can be converted to a hexahedral mesh, but the results are generally not acceptable, as the resulting mesh is not regular enough. Our template-based approach to the creation of volumetric hexahedral meshes is shown in Figure 6.

After a rough contouring of the mesh, the centroids of the contours on each slice are first localized, thus creating a "spine" curve. A generic volumetric mesh is then generated by replication along this curve (see Figure 6). After a coarse registration, the generic mesh is projected onto the surface extracted from the CT data set using the TrueGrid ${ }^{4}$ meshing package. The resulting mesh can be refined by smoothing and interpolation. Dynamic simulations are run after the boundary conditions are specified using the NIKE3D Finite Element Code developed at LLNL. Let us point out that this approach was not designed to handle highly asymmetrical objects. In that case, the registration between the extracted surface and the generic mesh is not precise enough to yield an accurate volumetric mesh. A solution consists in meshing separately different parts of an object and then computationally glue the different meshes together. Figure 7 shows the result of this template-based strategy on a metacarpal bone.
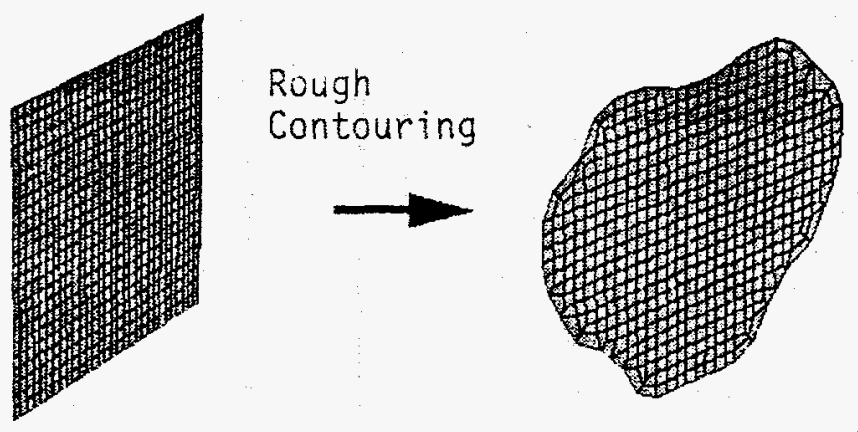

Replication along spine
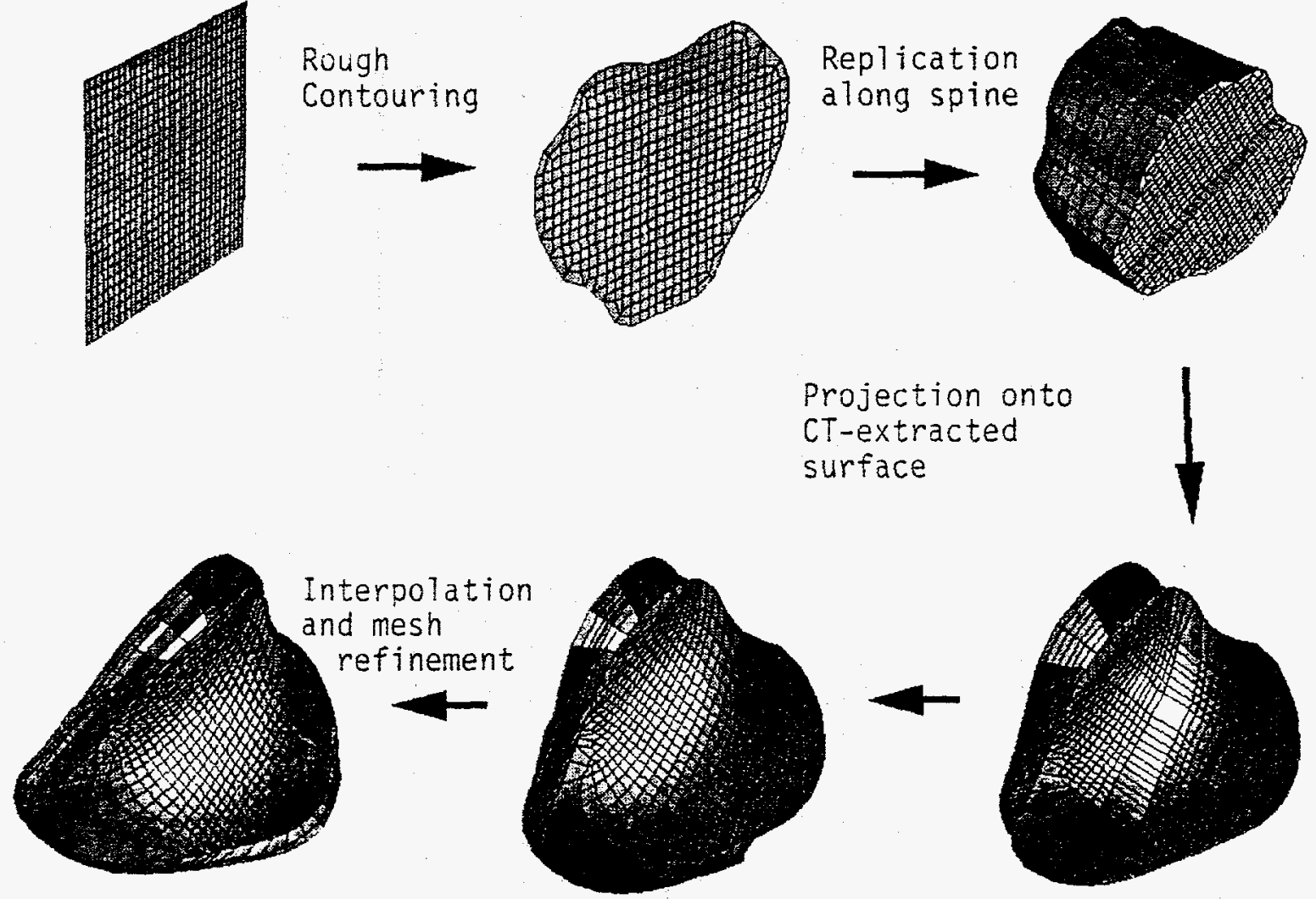

Figure 6: Overview of the template-based meshing approach (see text for details).

$+\mathrm{XYZ}$ Solutions Inc., Livermore, CA. 


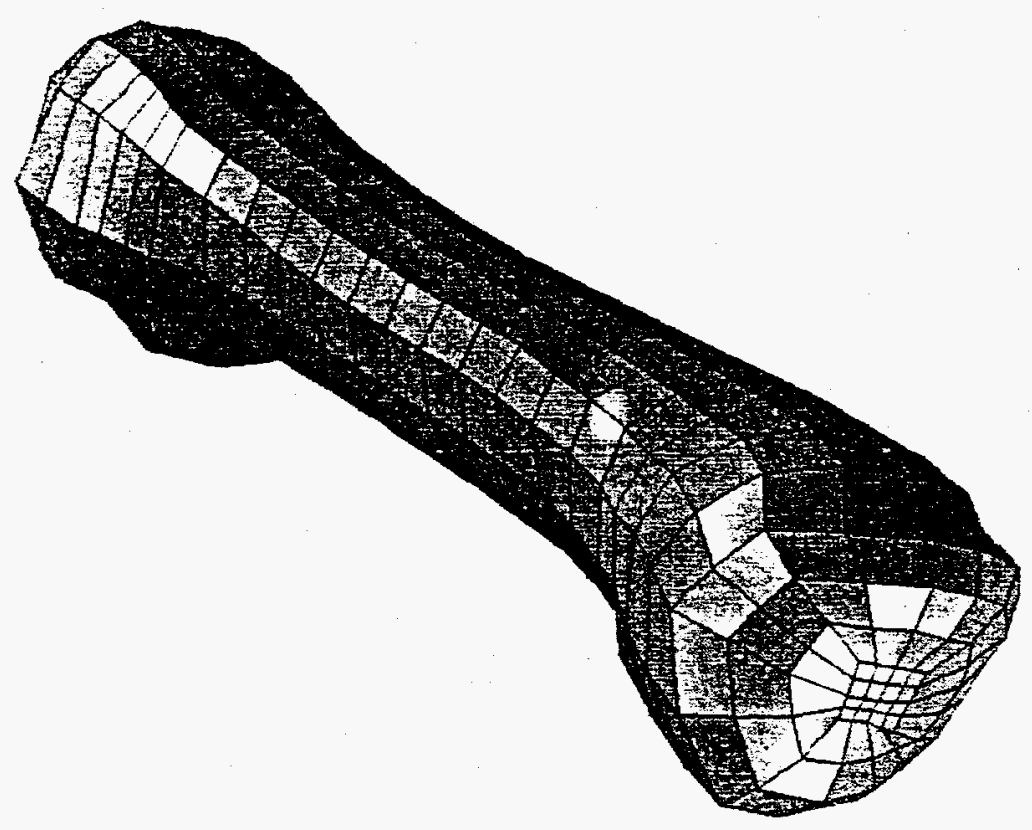

Figure 7: Volumetric mesh of a metacarpal bone (see text for details).

\section{CONCLUSION}

Our major goal in this work was to create accurate Finite Element meshes from 3D $\mathrm{X}$-ray $\mathrm{CT}$ data sets, and to minimize the amount of human interaction required. We described each of the steps of the data flow, namely the preprocessing approaches and tomographic reconstruction, the segmentation and surface extraction, as well as a templatebased mesh generation. The results obtained in a biomechanics application were detailed. We expect in the future to generalize this work to investigate industrial parts. Practical experiments have also showed the need for a decimation and smoothing of the surface mesh extracted from the CT volumes. We are considering as well an alternative solution to the meshing problem which would rely on an intermediate $\mathrm{CAD}$ model; this technique would by construction solve the surface smoothing problem, although it requires a fair amount of interaction which would limit its applicability.

\section{ACKNOWLEDGMENTS}

The authors would like to thank Dan Schneberk for the useful help and suggestions concerning the computational part of this work, Earl Updike for acquiring the data, and all the other members of the NonDestructive Evaluation Section that have contributed to various technical discussions. Discussions with Elaine Ashby on various biological aspects also helped us during this study.

\section{REFERENCES}

1. G.A. Mohr and F. Little, "Effective 3D Geometry Extraction and Reverse CAD Modeling", Review of Progress in QNDE, vol. 14, pp. 651-656, 1994.

2. J.H. Stanley, R.N. Yancey, Q. Cao and N.J. Dusaussoy, "Reverse Engineering and Rapid Prototyping for Solid Freeform Fabrication", SPIE vol. 2455, pp. 306-311, 1995. 
3. J. Canny, "A Computational Approach to Edge Detection", IEEE Transactions on Pattern Analysis and Machine Intelligence, vol. 8, n. 6, pp. 679-697, 1986.

4. L. Vincent, "Morphological Grayscale Reconstruction in Image Analysis: Applications and Efficient Algorithms", IEEE Transactions on Image Processing, vol. 2, n. 2, pp. 176201, April 1993.

5. L. Vincent and P. Soille, "Watersheds in Digital Spaces: An Efficient Algorithm Based on Immersion Simulations", IEEE Transactions on Pattern Analysis and Machine Intelligence, vol. 13, n. 6, pp. 583-598, June 1991.

6. W.E. Lorensen and H.E. Cline, "Marching Cubes: a High Resolution Surface Extraction Algorithm", Computer Graphics, vol. 21, n. 3, pp. 163-169, 1987.

7. W.J. Schroeder, J.A. Zarge and W.E. Lorensen, "Decimation of Large Triangle Meshes", Computer Graphics (SIGGRAPH 92), vol. 26, n. 2,pp. 65-70, 1992.

8. B. Geiger, "Three-Dimensional Modeling of Human Organs and its Application to Diagnosis and Surgical Planning", Ph.D. Thesis, Ecole des Mines de Paris, 1993.

9. P.M. Finnigan, A.F. Hathaway, W.E. Lorensen, I.J. Connell, V.N. Parthasarathy and J. B. Ross, "CATFEM - Computer Assisted Tomography and Finite Element Modeling", Control and Dynamic Systems, vol. 49, pp. 298-337, 1991. 